\title{
Comparison and Optimization of Multiplexed Quantum Dot-Based Immunohistofluorescence
}

\author{
Donghai Huang ${ }^{1,2}$, Xianghong Peng ${ }^{1}$, Ling Su${ }^{1}$, Dongsheng Wang ${ }^{1}$, Fadlo R. Khuri ${ }^{1}$, Dong M. Shin ${ }^{1}$, and \\ Zhuo (Georgia) Chen $^{1}(\bowtie)$ \\ ${ }^{1}$ Department of Hematology and Medical Oncology, Winship Cancer Institute, Emory University School of Medicine, Atlanta, Georgia \\ 30322, USA \\ ${ }^{2}$ Department of Otolaryngology-Head and Neck Surgery, Xiang-Ya Hospital, Central South University, Changsha, Hunan 410008, China \\ Received: 25 October 2009 / Revised: 25 November 2009 / Accepted: 25 November 2009 \\ (C) The Author(s) 2010. This article is published with open access at Springerlink.com
}

\begin{abstract}
Nanoparticle quantum dots (QDs) are ideal materials for multiplexed biomarker detection, localization, and quantification. Both direct and indirect methods are available for QD-based immunohistofluorescence (QD-IHF) staining; the direct method, however, has been considered laborious and costly. In this study, we optimized and compared the indirect QD-IHF single staining procedure using QD-secondary antibody conjugates and QD-streptavidin conjugates. Problems associated with sequential multiplex staining were identified quantitatively. A method using a QD cocktail solution was developed allowing simultaneous staining with three antibodies against E-cadherin, epidermal growth factor receptor and $\beta$-catenin in formalin-fixed and paraffin-embedded (FFPE) tissues. The expression of each biomarker was quantified by using the cocktail and the sequential methods. Comparison of the two methods demonstrated that the cocktail method provided more consistent and stable QD signals for each multiplexed biomarker than the sequential method, and provides a convenient tool for multiplexing biomarkers in both research and clinical applications.
\end{abstract}

\section{KEYWORDS}

Quantum dots, immunohistofluorescence, nanocrystals, spectral imaging

\section{Introduction}

In recent years, nanotechnology has developed rapidly and been used in molecular detection, imaging, diagnostics, and therapeutics in the cancer field [1,2]. Quantum dots (QDs) are nanoscale particles made from inorganic semiconductors that can produce different fluorescence signals depending on their size and components. Compared with organic dyes, QDs have superior signal brightness and photostability, relatively long excited-state lifetime, and optimized

Address correspondence to gzchen $@$ emory.edu signal-to-background ratios [3]. QDs can be covalently linked to biological molecules such as peptides, proteins, and nucleic acids, as well as streptavidin $[4,5]$. Due to their long excitation time and narrow emission spectra, QDs can be excited simultaneously through one appropriate excitation source. Together these properties render QDs ideal for multiplexed biological imaging and they have been used for both molecular and cellular labeling [3-7].

Many researchers reported that QDs can immunostain more than three biomarkers in formalin-fixed 
paraffin-embedded (FFPE) tissues using QD-based immunohistofluorescence (QD-IHF) [8-11]. To date, several different staining procedures have been utilized, including direct and indirect staining, such as QDs linked to primary antibodies and QDs linked to secondary antibodies or streptavidin, respectively [9, 10, 12, 13]. Although the direct staining method (QDs linked directly to a primary antibody) is straightforward, the affinity of some primary antibodies may be reduced during the QD conjugation process. The conformation and function of the primary antibody may be changed and its binding properties are likely altered by covalent modifications at either $-\mathrm{NH}_{2}$ or - $\mathrm{COOH}$ sites $[9,14]$. Furthermore, the reagent costs are considerable because each conjugation reaction requires up to $300 \mu \mathrm{g}$ of antibody (Invitrogen protocol) and the yield of QD-antibody conjugates is usually low. Since each primary antibody is covalently conjugated to just one type of $\mathrm{QD}$, changing the antibody for a particular QD probe is not possible once the conjugation is completed. Many researchers have abandoned the direct staining method since these problems can be avoided by indirect QD staining methods.

The main advantages of indirect QD staining are its flexibility, lower costs, and the reduced constraints on primary antibodies. Although many studies have described detailed protocols for tissue specimen preparation, multicolor QD staining, and image processing $[8,9,15]$, these have not provided detailed discussion or quantitative analysis of the optimization of their multiplexed biomarker staining procedures. In this study, we compared multiple QD staining in a sequential order with that in a simultaneous combination while using different methods-QD-secondary antibody conjugates and QD-streptavidin conjugates. QD staining of three biomarkers, epidermal growth factor receptor (EGFR), E-cadherin (E-cad), and $\beta$-catenin ( $\beta$-cat), was examined and quantitatively evaluated with these staining methods for each of the tested biomarkers.

\section{Experimental}

Using an institutional review board-approved consent for tissue acquisition, tissue samples for this study were obtained from surgical specimens from patients who were diagnosed at Emory University Hospital with squamous cell carcinoma of the head and neck (SCCHN). After a routine process to generate FFPE samples, the blocks were sectioned to $4 \mu \mathrm{m}$ each and mounted on coated slides. Each sample was analyzed by hematoxylin and eosin (H\&E) staining. Before QD-IHF staining, we confirmed that the primary antibodies were suitable for immunohistochemistry (IHC) and also selected FFPE samples which were strongly positive for staining of the primary antibodies as positive control slides.

We selected mouse anti-human E-cad, rabbit antihuman EGFR, and goat anti-human $\beta$-cat as the primary antibodies, since these antigens are strongly expressed in SCCHN tissues. We found previously that expression and localization of E-cad and EGFR correlated with metastasis and poor prognosis [16]. QD-secondary antibody conjugates (QD-2nd $\mathrm{Ab}$ ) and QD-streptavidin conjugates (QD-streptavidin) were selected as follows: Qdots ${ }^{\circledR} 565$ goat $\mathrm{F}\left(\mathrm{ab}^{\prime}\right)_{2}$ anti-mouse immunoglobulin G (IgG) conjugate, Qdots ${ }^{\circledR} 605$ goat $\mathrm{F}\left(\mathrm{ab}^{\prime}\right)_{2}$ anti-rabbit IgG conjugate, Qdots ${ }^{\circledR} 655$ rabbit $\mathrm{F}\left(\mathrm{ab}^{\prime}\right)_{2}$ anti-goat IgG conjugate, and Qdots ${ }^{\circledR}$ streptavidin conjugate $(565,605,655)$.

The QD-IHF procedure with single QD-2nd Ab was carried out as follows (shown in the cartoon in Fig. 1(a)). After deparaffinization and rehydration, antigen retrieval was performed by heating with citric acid $(10 \mathrm{mmol} / \mathrm{L}, \mathrm{pH} 6.0)$ in a microwave to $95{ }^{\circ} \mathrm{C}$ for $10 \mathrm{~min}$. The tissue slides were blocked with $5 \%$ normal goat serum (Dako) for $10 \mathrm{~min}$ before the primary antibody incubation (E-cad 1:2000 dilution, EGFR 1:150 dilution, or $\beta$-cat 1:2000 dilution) for $1 \mathrm{~h}$ at $37^{\circ} \mathrm{C}$. Following three washes with phosphate-buffered saline (PBS) (5 min each), the slides were incubated with QD [QD565 goat $\mathrm{F}\left(\mathrm{ab}^{\prime}\right)_{2}$ anti-mouse IgG conjugate, QD605 goat $\mathrm{F}\left(\mathrm{ab}^{\prime}\right)_{2}$ anti-rabbit IgG conjugate, or QD655 rabbit $\mathrm{F}\left(\mathrm{ab}^{\prime}\right)_{2}$ anti-goat IgG conjugate, accordingly] in $6 \%$ bovine serum albumin (BSA) for $1 \mathrm{~h}$ at $37^{\circ} \mathrm{C}$. After washing three times with PBS, the nuclei were counterstained with 4',6-diamidino-2phenylindole (DAPI) (Invitrogen, Carlsbad, CA, USA). The slides were mounted with Cytoseal ${ }^{\mathrm{TM}} 60$ mounting medium (Richard-Allan Scientific, MI). 

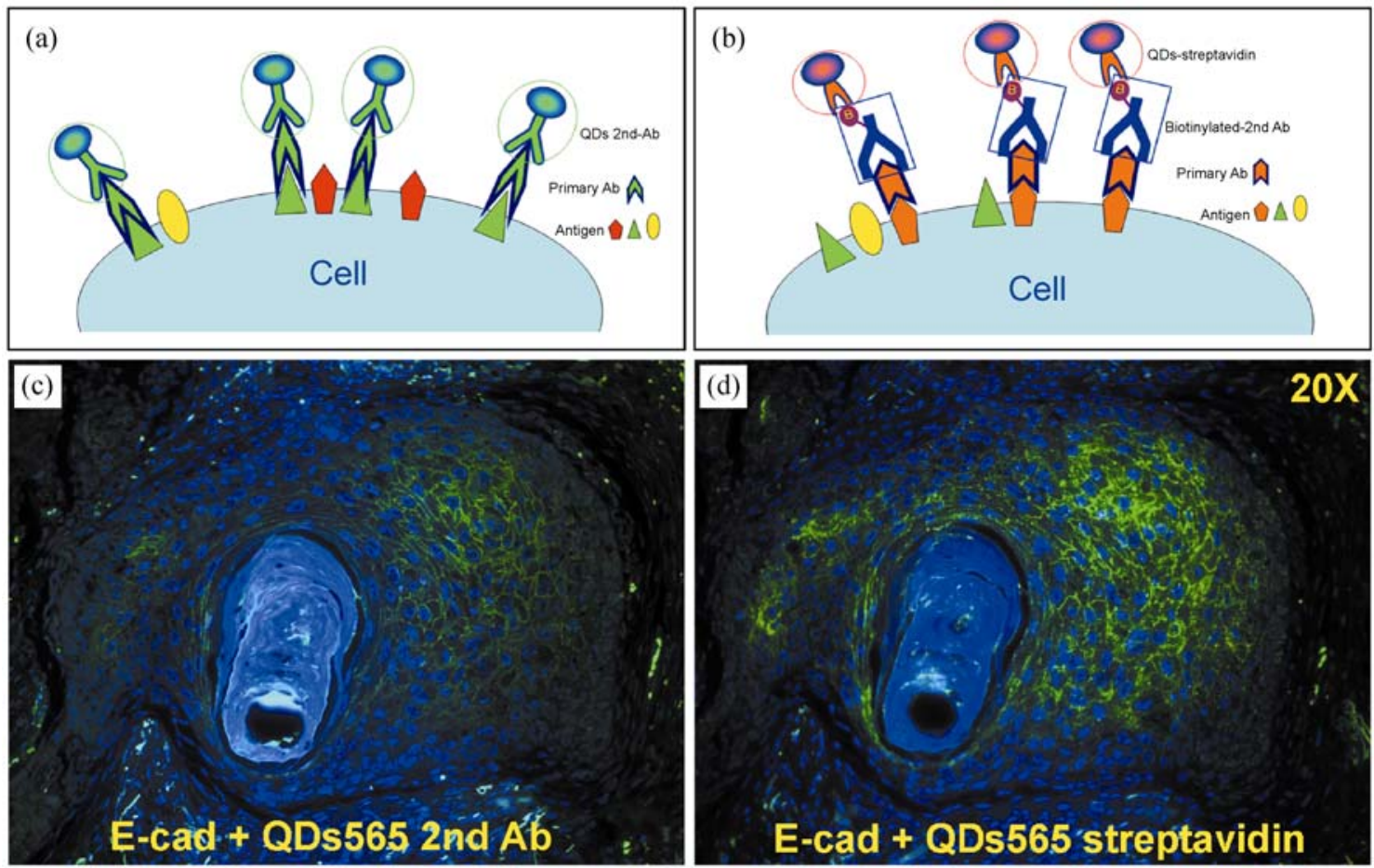

(e)

Signal comparison between QDs-2nd Ab and

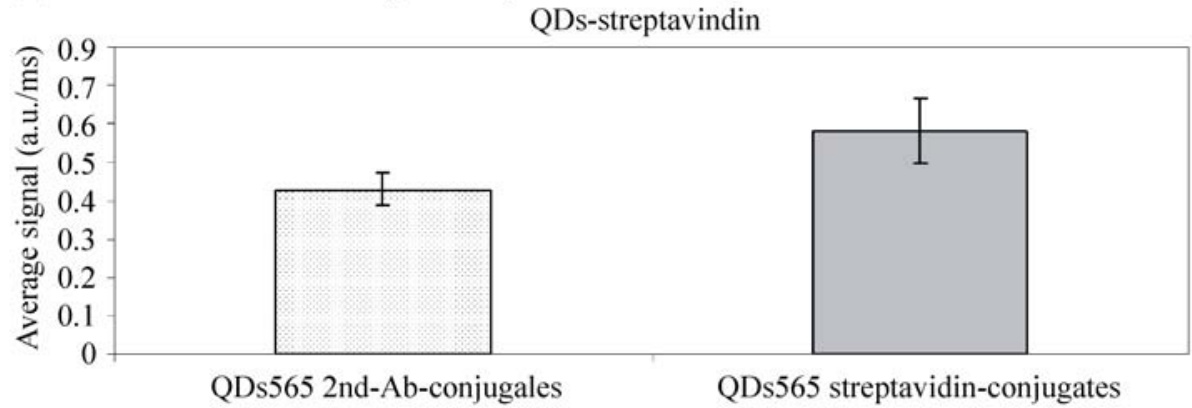

Figure 1 Comparison of single QD-IHF staining using QD-2nd Ab with QD-streptavidin: (a) cartoon showing single QD-IHF staining with QD-2nd Ab conjugates; (b) cartoon showing single QD-IHF staining with QD-streptavidin conjugates; (c) RGB image of E-cad QD-IHF staining with QD565-2nd Ab; (d) RGB image of E-cad QD-IHF staining with QD565-streptavidin; (e) signal intensity comparison between QD-2nd Ab and QD-streptavidin

For QD-IHF staining with QD-streptavidin (shown in the cartoon in Fig. 1(b)), slides were prepared as above. After the primary antibody incubation, slides were incubated with biotinylated $2 \mathrm{nd} \mathrm{Ab}$ for $20 \mathrm{~min}$ at room temperature (RT), and washed three times with PBS (5 min each). Slides were incubated with QD565-, QD605-, or QD655-streptavidin (1:100) in $6 \%$ BSA for $1 \mathrm{~h}$ at $37^{\circ} \mathrm{C}$ and washed three times with PBS (5 min each). After nuclei counterstaining and mounting, the slides were kept in the dark at $4{ }^{\circ} \mathrm{C}$ for visualizing and quantifying. Mouse, rabbit or goat
IgG was used as a negative control.

For sequential QD-IHF staining with QD-streptavidin, after the first primary antibody E-cad incubation (1:2000 dilution), the slides were incubated with the biotinylated $2 \mathrm{nd} \mathrm{Ab}$ for $20 \mathrm{~min}$ at room temperature and washed three times with PBS (5 min each). Slides were then incubated with QD565-streptavidin (1:100) in $6 \%$ BSA for $1 \mathrm{~h}$ at $37^{\circ} \mathrm{C}$ and washed three times with PBS (5 min each). After staining the first biomarker with QDs, the staining procedure was repeated from the blocking step, except the primary antibody and 
QD conjugate were replaced with second biomarker EGFR (1:150) and QD605-streptavidin (1:100), respectively. Then the slides were mounted after nuclear counterstaining. For QD signal comparison, we also switched the staining sequence from EGFR with QD565-streptavidin staining as the first step to E-cad with QD605-streptavidin staining as the second. Mouse and rabbit IgG were used as a negative control.

For the cocktail staining method, we chose primary antibodies of distinct species origins, including mouse anti-human E-cad, rabbit anti-human EGFR, and goat anti-human $\beta$-cat. Therefore, for QD-2nd Abs, we selected QD565 goat $\mathrm{F}\left(\mathrm{ab}^{\prime}\right)_{2}$ anti-mouse IgG, QD605 goat $\mathrm{F}\left(\mathrm{ab}^{\prime}\right)_{2}$ anti-rabbit IgG, and QD655 rabbit $\mathrm{F}\left(\mathrm{ab}^{\prime}\right)_{2}$ anti-goat IgG, respectively. After preparation steps, the slides were incubated with the three primary antibodies against E-cad (1:2000), EGFR (1:150), and $\beta$-cat (1:2000) simultaneously for $1 \mathrm{~h}$ at $37^{\circ} \mathrm{C}$. After washing three times with PBS, the three QD-2nd Abs in a cocktail solution at 1:100 dilution were added to the slides with further incubation for $1 \mathrm{~h}$ at $37^{\circ} \mathrm{C}$. Slides were washed three times in PBS, counterstained, mounted, and stored as described above. For the sequential method (shown in the cartoon in Fig. 4(a)(ii)), the additional biomarker $\beta$-cat was stained by incubation with QD655-streptavidin following staining for E-cad with QD565-streptavidin and EGFR with QD605-streptavidin as above. The IgG with the same host species as the $2 \mathrm{nd} \mathrm{Ab}$ was used as a negative control.

An Olympus microscope IX71 with CRi Nuance spectral imaging and quantifying system (CRi Inc., Woburn, MA, USA) was used to observe and quantify the QD signals. All cubed image files were collected from the FFPE tissue slides at $10-\mathrm{nm}$ wavelength intervals from 500 to $800 \mathrm{~nm}$ with an auto exposure time at $200 \times$ magnification. Taking the cube with a long wavelength bandpass filter allowed transmission of all emission wavelengths above $450 \mathrm{~nm}$. Both mixed and separated QD images were established after determining the QD spectral library and unmixing the cube. Background and auto-fluorescence were removed for accurate quantification of each QD signal. For comparison of the QD signals, we defined the measurement threshold as the same in each case.
An arbitrary unit (a.u.) was defined as the average fluorescence signal intensity per exposure time (ms), in which the exposure time was optimized so that the differences in fluorescence intensity among QDs were minimized and the same exposure time could be used for all QDs. These values were obtained directly from the Nuance software. Ten randomly selected fields in each sample slide were used for quantification. Data are presented as a mean of ten readings with the standard deviation (S. D.).

\section{Results and discussion}

We used the quantification results to evaluate the working conditions. It was found that (1) the same antigen retrieval method as used in IHC also performed well in QD-IHF staining of FFPE samples; (2) the optimized working conditions for primary antibodies in IHC also worked well for QD-IHF; (3) incubation of the QD-conjugates from Invitrogen at $10-20 \mathrm{nmol} / \mathrm{L}$ and $37^{\circ} \mathrm{C}$ for $1 \mathrm{~h}$ was sufficient to reach a balance of the maximum staining effect with minimized non-specific binding. Non-specific binding increased when increasing either the concentration or the incubation time of the QD-conjugates, suggesting that $10-20 \mathrm{nmol} / \mathrm{L}$ of QD-conjugate may be a saturating level for staining. There was almost no significant difference in the intensity of QD signal when the concentration of QD-conjugates reached $20 \mathrm{nmol} / \mathrm{L}$, but the non-specific binding increased directly (data not shown), suggesting that the QD binding was saturated at $20 \mathrm{nmol} / \mathrm{L}$; (4) multiple PBS washing up to three times did not reduce the QD signal intensity. The effects of other washing buffers, such as PBS with Tween-20 (PBS-T) or Tris-buffered saline with Tween-20 (TBS-T), were similar to that of PBS. The antigen retrieval method, dilution, and incubation condition of the antibody are the main factors that affect the results of immunostaining FFPE tissues. Most researchers use the same retrieval method and incubation conditions for primary antibodies when conducting IHC and IHF stained with QDs. There are several issues that we have to address before immunostaining with QD-bioconjugates: (1) do the optimized working conditions for IHC work well for 
QD-IHF? (2) how can we control the dilution ratio for QD-conjugates and the incubation conditions to obtain a balance between an optimal signal and minimized non-specific binding? (3) how can we optimize the QD-IHF staining procedure, especially in multiple staining? These potential problems have been addressed using the quantification data in our studies.

Figure 1 shows that the signal when staining with QD-2nd Ab was lower than that with QD-streptavidin (Figs. 1(c), 1(d)). The quantification results also showed that the average intensity from QDstreptavidin staining was 1.36-1.73-fold greater than that from QD-2nd Ab staining (Fig. 1(e)). The staining with QD-streptavidin had some amplification effect.

For multiplex QD staining, the sequential staining method is used by most researchers [8, 12]. To investigate whether the intensity of the QD signal after the first step changes or not after the subsequent biomarker staining and many washing steps, we initially tested sequential QD-IHF staining of E-cad with QD565-streptavidin followed by EGFR with QD605-streptavidin, and then altered this sequence. The staining signals from the two experiments were quantified and compared. It was found that the QD intensity of E-cad staining when stained first was $0.104 \pm 0.050$ compared with $0.534 \pm 0.132$ when stained second (Fig. 2(a)). Similarly, the intensity of EGFR staining when stained first was $0.189 \pm 0.104$ compared with $0.565 \pm 0.098$ when stained second (Fig. 2(b)). This result indicated that the intensity of the first QD signal after the initial biomarker staining was reduced following the second blocking and washing steps.

In order to achieve the best staining of each biomarker using the QD-IHF sequential method, the selection of QDs may be considered. Our study has demonstrated that the intensity of QDs from Invitrogen at the same concentration was different and varied in the order QD655 > QD605 > QD565 (Fig. 3). In theory, the QD with highest intensity is recommended to be used in the first step to offset the decreasing signal when staining with QD-IHF in a sequential manner.

In order to avoid the decrease in signal observed with sequential staining, we applied three mixed primary antibodies with distinct species origins to the tissue slides and then incubated the relevant QD-2nd
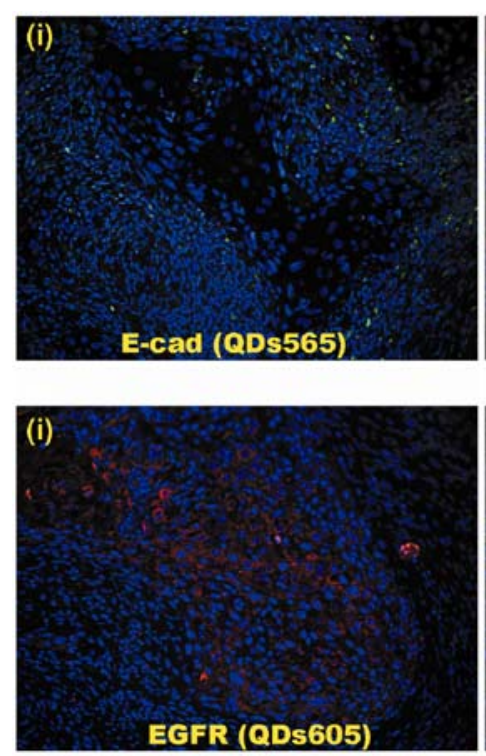

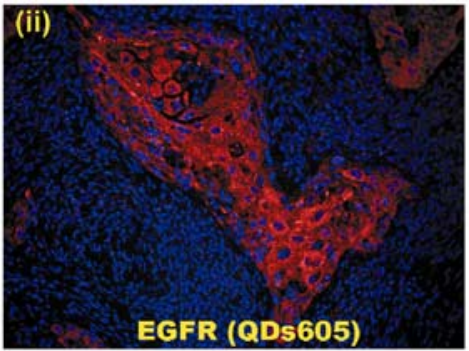

(a)

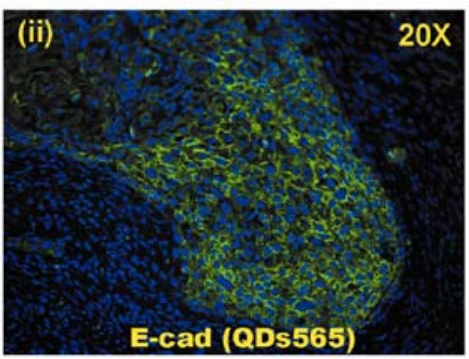

(b)
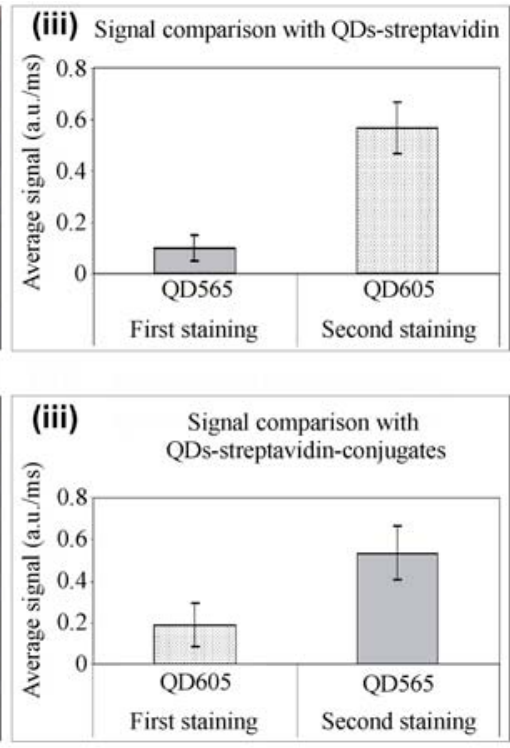

Figure 2 Comparison of the first signal with the second signal in a sequential QD-IHF staining. (a) E-cad with QD565-streptavidin as the first biomarker and EGFR with QD605-streptavidin as the second. (b) EGFR with QD605-streptavidin as the first biomarker and E-cad with QD565-streptavidin as the second. (i) unmixed first signal; (ii) unmixed second signal; (iii) quantification comparison between these two biomarkers 


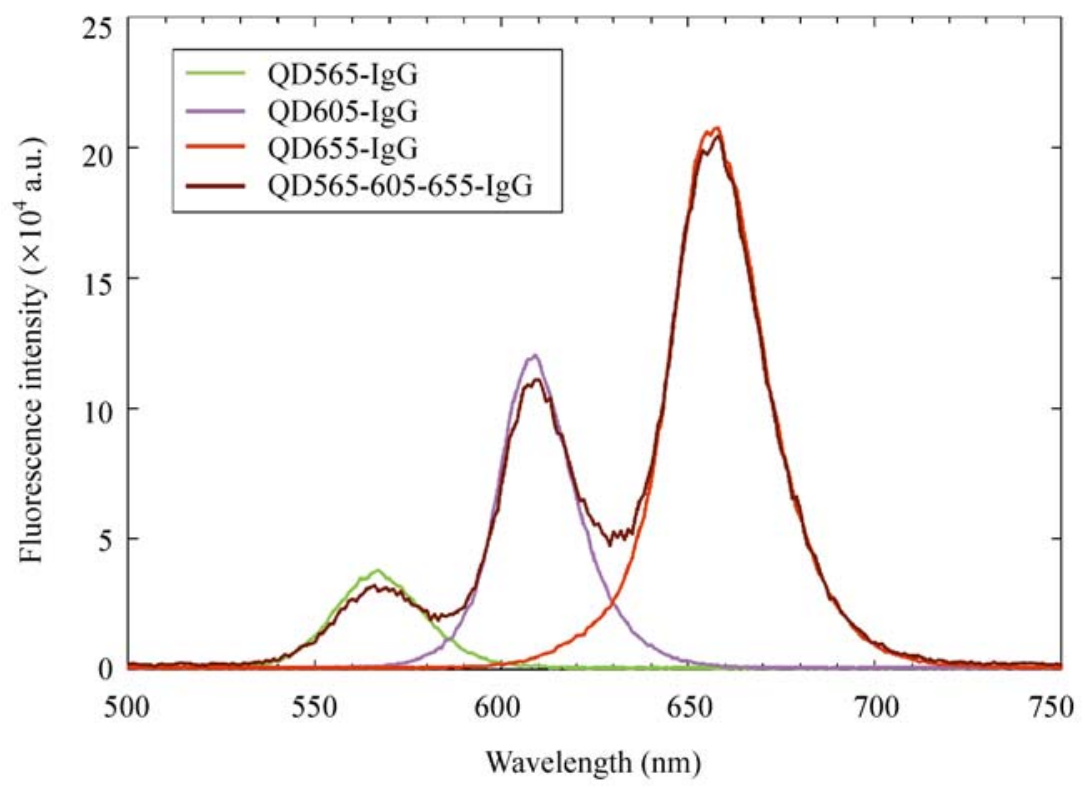

Figure 3 Comparison of QD emission intensity in single QD and cocktail PBS solutions. Fluorescence intensity of each of the three QDs was detected by QuantaMaster ${ }^{\mathrm{TM}}$ UV VIS, (Photon Technology International, Birmingham, NJ)

Abs in a cocktail solution in order to make the IHF staining efficient and simple [shown in the cartoon in Fig. 4(a)(i)]. The level of each QD signal obtained from the cocktail method was quantified and compared to that obtained with the sequential method. It was found that all of the QD signals obtained by the QD-IHF cocktail method were consistent (Fig. 4(b)). The intensities of E-cad, EGFR, and $\beta$-cat were $0.318 \pm$ $0.015,0.309 \pm 0.034$, and $0.362 \pm 0.036$, respectively (Fig. 4(d)). In contrast, the signals from the sequential staining method were not consistent (Fig. 4(c)). Intensities of the second and the third signals were, respectively, 1.57-2.20- and 5.80-8.24-fold higher than the first signal (Fig. 4(d)).

Because the properties of nanocrystals are highly dependent on the surface environment, whether the stability with respect to the optical emission peak maximum and color purity of the QDs in such a cocktail solution may be changed is always a consideration. As recommended by the QD manufacturer, Invitrogen Cooperation, we diluted the three QDs with 6\% BSA in PBS solution, and tested the signal intensity of the QDs either singly or in a cocktail solution using a spectrofluorimeter [QuantaMaster ${ }^{\mathrm{TM}}$ UV VIS, Photon Technology
International (PTI)]. Our study confirmed that the QD signals in PBS appeared at the expected wavelength with reasonable sensitivity (Fig. 3). Furthermore, the fluorescence intensity of each QD was not altered in the cocktail solution when compared to the single QD solution (Fig. 3).

One of the drawbacks of the cocktail method is that it can be challenging to find more than four primary antibodies with distinct species origins for simultaneous IHF staining, which limits the use of this method to not more than four biomarkers. In the case of multiplexing more than four biomarkers, the cocktail plus the sequential method may be applied.

\section{Conclusions}

We have demonstrated that the signal intensities using the QD-streptavidin-based staining method were higher than those with QD-2nd Ab. QD staining signals using the cocktail method were more consistent and stable than those obtained using the sequential method. In order to achieve the optimal signal for each biomarker in a QD-IHF multiplexed staining procedure, the staining method selection and QD intensity should be considered. 


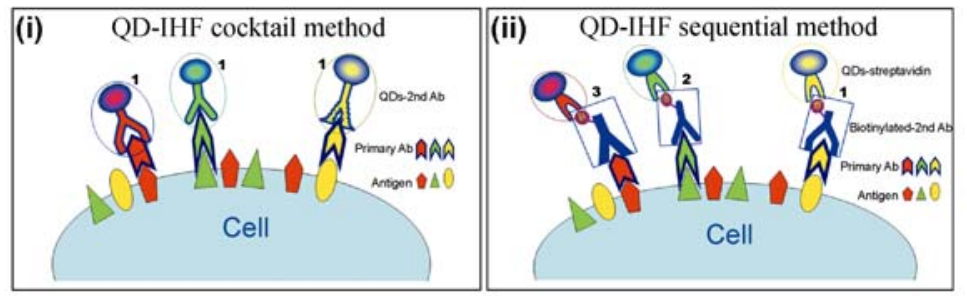

(a)
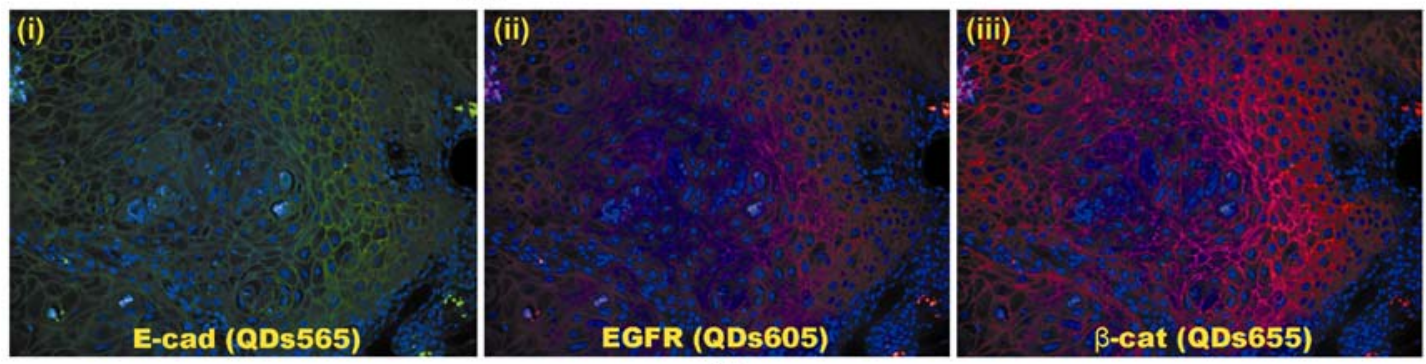

(b)
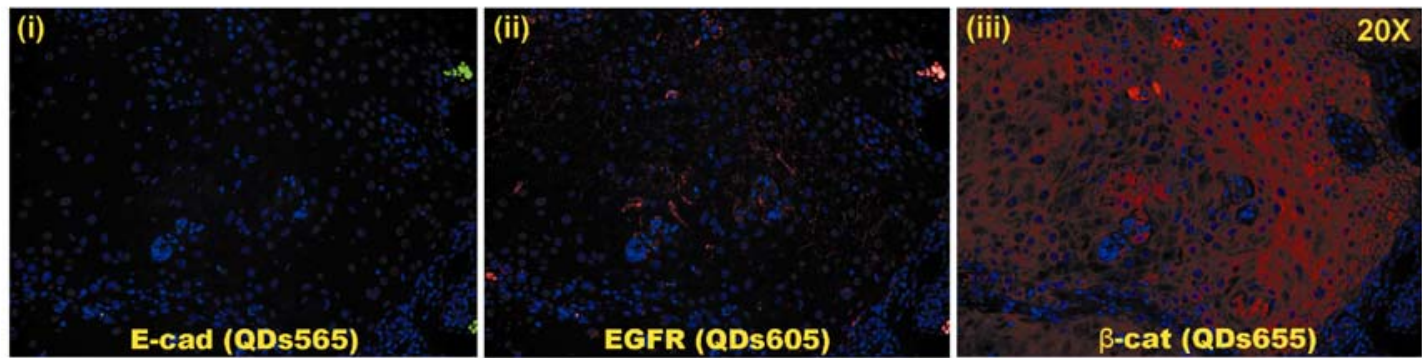

(c)

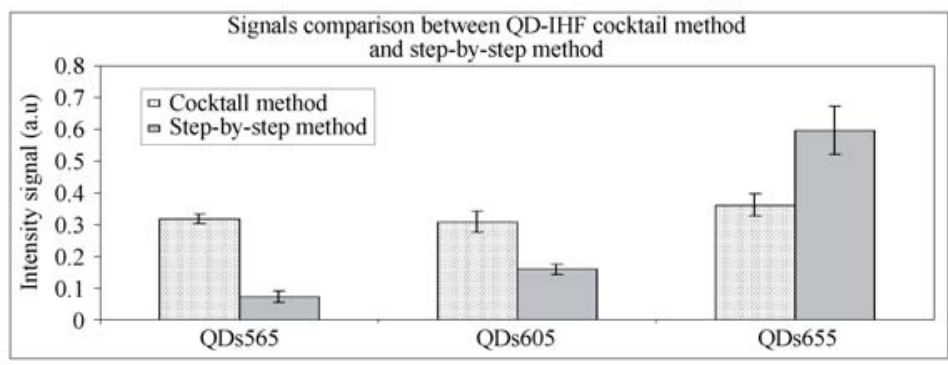

(d)

Figure 4 Comparison of the QD-IHF cocktail method with the sequential method. (a) (i) Cartoon showing cocktail QD-IHF staining with QD-2nd Ab conjugates, "1" illustrates the addition of different QDs simultaneously; (ii) cartoon showing sequential QD-IHF staining with QD-streptavidin conjugates, “1,2,3” represent the addition of QDs at different steps. (b) Cocktail QD-IHF staining of E-cad + EGFR + $\beta$-cat with QD565 + 605 + 655 2nd Ab-conjugates. (c) Sequential QD-IHF staining of E-cad, EGFR, and $\beta$-cat with QD565-, 605-, and 655-streptavidin conjugates, respectively: (i) unmixed E-cad (QD565) signal; (ii) unmixed EGFR (QD605) signal; (iii) unmixed $\beta$-cat (QD655) signal. (d) Quantified signal comparison between these two methods

\section{Acknowledgements}

This study was supported by DOD Grant W81XWH07-1-0306 Project 5 and GCC Distinguished Scholar Award to $Z(G)$. C. We thank Dr. Ximei Qian for testing QD fluorescence intensity and Dr. Anthea Hammond for her critical reading of the manuscript.

\section{References}

[1] Ferrari, M. Cancer nanotechnology: Opportunities and challenges. Nat. Rev. Cancer 2005, 5, 161-171.

[2] Nie, S. M.; Xing, Y.; Kim, G. J.; Simons, J. W. Nanotechnology applications in cancer. Ann. Rev. Biomed. Eng. 2007, 9, 257-288. 
[3] Smith, A. M.; Gao, X. H.; Nie, S. M. Quantum dot nanocrystals for in vivo molecular and cellular imaging. Photochem. Photobiol. 2004, 80, 377-385.

[4] Whaley, S. R.; English, D. S.; Hu, E. L.; Barbara, P. F.; Belcher, A. M. Selection of peptides with semiconductor binding specificity for directed nanocrystal assembly. Nature 2000, 405, 665-668.

[5] Chan, W. C. W.; Nie, S. M. Quantum dot bioconjugates for ultrasensitive nonisotopic detection. Science 1998, 281, 2016-2018.

[6] Wu, X. Y.; Liu, H. J.; Liu, J. Q.; Haley, K. N.; Treadway, J. A.; Larson, J. P.; Ge, N. F.; Peale, F.; Bruchez, M. P. Immunofluorescent labeling of cancer marker Her2 and other cellular targets with semiconductor quantum dots. Nat. Biotechnol. 2003, 21, 41-46.

[7] Gao, X. H. Multifunctional quantum dots for cellular and molecular imaging. Conf. Proc. IEEE Eng. Med. Bio. Soc. 2007, 2007, 524-525.

[8] Sweeney, E.; Ward, T. H.; Gray, N.; Womack, C.; Jayson, G.; Hughes, A.; Dive, C.; Byers, R. Quantitative multiplexed quantum dot immunohistochemistry. Biochem. Biophys. Res. Commun. 2008, 374, 181-186.

[9] Xing, Y.; Chaudry, Q.; Shen, C.; Kong, K. Y.; Zhau, H. E.; Chung, L. W.; Petros, J. A.; O'Regan, R. M.; Yezhelyev, M. V.; Simons, J. W.; Wang, M. D.; Nie, S. M. Bioconjugated quantum dots for multiplexed and quantitative immuno- histochemistry. Nat. Protoc. 2007, 2, 1152-1165.

[10] Fountaine, T. J.; Wincovitch, S. M.; Geho, D. H.; Garfield, S. H.; Pittaluga, S. Multispectral imaging of clinically relevant cellular targets in tonsil and lymphoid tissue using semiconductor quantum dots. Modern Pathol. 2006, 19, 1181-1191.

[11] True, L. D.; Gao, X. H. Quantum dots for molecular pathology: Their time has arrived. J. Mol. Diagn. 2007, 9, 7-11.

[12] Shi, C.; Zhou, G.; Zhu, Y.; Su, Y.; Cheng, T.; Zhau, H. E.; Chung, L. W. K. Quantum dots-based multiplexed immunohistochemistry of protein expression in human prostate cancer cells. Eur. J. Histochem. 2008, 52, 127-133.

[13] Yang, D. Z.; Chen, Q. F.; Wang, W. X.; Xu, S. K. Direct and indirect immunolabelling of HeLa cells with quantum dots. Luminescence 2008, 23, 169-174.

[14] Resch-Genger, U.; Grabolle, M.; Cavaliere-Jaricot, S.; Nitschke, R.; Nann, T. Quantum dots versus organic dyes as fluorescent labels. Nat. Methods 2008, 5, 763-775.

[15] Tholouli, E.; Sweeney, E.; Barrow, E.; Clay, V.; Hoyland, J. A.; Byers, R. J. Quantum dots light up pathology. J. Pathol. 2008, 216, 275-285.

[16] Muller, S.; Su, L.; Tighiouart, M.; Saba, N.; Zhang, H. Z.; Shin, D. M.; Chen, Z. G. Distinctive E-cadherin and epidermal growth factor receptor expression in metastatic and nonmetastatic head and neck squamous cell carcinoma: Predictive and prognostic correlation. Cancer 2008, 113, 97-107. 